\title{
Discussão sobre a Adaptabilidade em Ambientes U-learning baseada em Estilos de Aprendizagem
}

\author{
Luma da Rocha Seixas¹, Ivanildo José de Melo Filho ${ }^{1,2}$, Alex Sandro Gomes ${ }^{1}$ \\ ${ }^{1}$ Centro de Informática - Universidade Federal de Pernambuco (UFPE) \\ Caixa Postal 7.851 - 54.740-540 - Recife - PE - Brazil \\ ${ }^{2}$ Instituto Federal de Pernambuco - Campus Belo Jardim (IFPE) \\ Av. Sebastião Rodrigues da Costa, S/N - CEP: 55155-730 - Belo Jardim - PE - Brazil \\ \{lrs3, asg\}@cin.ufpe.br, ivanildo.melo@belojardim.ifpe.edu.br
}

\begin{abstract}
This article aims to present and discuss with this community the adaptability process in the U-Learning environments. Such environments need to consider the identification of user needs, mobile technologies and the different learning situations that may occur. It is known that different environments make use of different recognition strategies adaptation process based on profiles. The literature emphasizes the limiting nature of this kind of recognition in these environments, mainly in and not consider the different learning styles.
\end{abstract}

Resumo. Este artigo tem como objetivo apresentar e discutir com esta comunidade o processo de adaptabilidade nos ambientes de U-Learning. Tais ambientes precisam considerar a identificação das necessidades do usuário, tecnologias móveis bem como as diferentes situações de aprendizagem que podem ocorrer. É sabido que diferentes ambientes fazem uso de diferentes estratégias de reconhecimento de processo de adaptação baseado em perfis. A literatura enfatiza o caráter limitante deste tipo de reconhecimento nesses ambientes, principalment e por não considerar os diferentes estilos de aprendizagem dos aprendizes.

\section{Introdução}

A possibilidade do acesso instantâneo a informações e a conteúdos tem fomentado o uso de tecnologias, fazendo com que as práticas em sala de aula busquem diferentes maneiras de incentivar um aprendizado contextualizado, motivado por demandas que podem ser específicas em função do conteúdo, do objetivo, bem como, da sua localização ou pelo tempo que se pretende alcançar.

Pesquisas tem apontado fortemente para uma aprendizagem individualizada e colaborativa na educação escolar (KONG et al., 2014). As salas de aula presenciais mantêm a sua importância na interação e socialização dos aprendizes. Contudo, as situações de aprendizagem fora da sala de aula tem superado as expectativas propostas pelo ensino tradicional e, consequentemente desempenham um papel importante na construção do conhecimento dos aprendizes (KONG et al., 2014). 
Chen e Li (2010) e Peng et al., (2008) evidenciam que os ambientes de aprendizagem ubíqua (u-learning) tem se posicionado como uma ferramenta com potencial no que diz respeito a adaptabilidade e flexibilidade para diferentes contextos de aprendizagem. Outras pesquisas relatam ainda significativa eficiência na aprendizagem nesses contextos (CHU et al., 2010; CHU, HWANG and TSAI, 2010; YANG, 2006). Barbosa (2007) enfatiza que nesses ambientes o modelo computacional deve atuar de forma invisível (background) e proativamente diante das necessidades dos aprendizes em suas atividades cotidianas, com base nas tecnologias, comunidades de aprendizagem e de interação social, nas redes de comunicação e nos demais serviços disponíveis.

Para Durán et al., (2014) ambientes u-learning incorporam um modelo de aprendizagem ad-hoc: aprendizagem adaptativa. Este modelo baseia-se na ideia de adaptar as metodologias de aprendizagem as formas de aprender dos alunos para proporcionar um conhecimento específico e personalizado quando necessário. Sendo assim, é possível compreender que a aprendizagem ubíqua busca combinar as vantagens da aprendizagem adaptativa, com os benefícios da computação ubíqua. E, ao contrário de modelos de aprendizagem desenvolvidos na última década e implementados por meio de uma arquitetura cliente-servidor, a aprendizagem ubíqua tem sido caracterizada por proporcionar maneiras intuitivas para identificar os conteúdos, serviços e colaboradores para uma aprendizagem no lugar e no momento adequado (BRANQUINHO et al., 2015).

No sentido de introduzir a adaptabilidade, iniciativas como Brusilovsky, Eklund e Schwarz (1998), Zakaria e Brailsford (1998) e Wu, Kort e Bra (2011) começaram a ser observadas nos últimos anos com o objetivo de personalizar o processo de aprendizagem. Em outras palavras, procuram proporcionar mecanismos personalizados para que o aprendiz possa selecionar caminhos dentro das possibilidades ofertadas pelo professor ou tutor (BRA e CALVI, 1998).

É preciso reconhecer que diversos são os sistemas que utilizam o perfil do aprendiz, porém, o uso deste para auxílio no processo de aprendizagem em um cenário ubíquo é recente (OGATA et al., 2005; BARBOSA, GEYER e BARBOSA, 2005; GASPERINI et al., 2004). Um ponto importante destacado por Aliança Neto, Neto, e Moreira (2014) refere-se a captura e manutenção eficaz do perfil do usuário, que de acordo com os autores não é uma atividade trivial. Sendo portanto, um dos problemas mais recorrentes neste processo a captura da informação.

Este artigo tem como propósito refletir a cerca de como ambientes de $u$-learning podem ser constituídos como uma alternativa para se adequar eficazmente às características dos aprendizes, principalmente em situações de contexto urbano. Dentro desse contexto de aprendizagem ubíqua, a adaptabilidade e a flexibilidade são pontos chaves para um melhor entendimento das reais necessidades dos aprendizes. Um ponto desafiador que permeia essa discussão concentra-se na percepção sobre o perfil dos aprendizes. Compreender o perfil do aprendiz, levando em consideração aspectos associados às suas características e o contexto ao qual ele encontra-se sinaliza como uma oportunidade a ser explorada nesse campo.

A organização desse documento é descrita a seguir: a seção 2 descreve trabalhos correlatos na comunidade científica brasileira. A seção 3 discute estudos que apontam o 
uso de estilos de aprendizagem como alternativa para personalizar os ambientes. As considerações finais são sintetizadas na seção 4 .

\section{Trabalhos correlatos}

$\mathrm{Na}$ aprendizagem ubíqua, a percepção sobre os elementos que envolvem o aprendiz transcende o virtual e alcança o mundo real (LEVIS et al., 2008). Um dos principais desafios nesse campo de pesquisa tem como ponto central o perfil do aprendiz ubíquo. Ao longo dos anos, pesquisas no âmbito nacional tem apresentado iniciativas com o suporte de ambientes de computação ubíqua, no intuito de promover uma aprendizagem personalizada. A seguir são apresentados 3 (três) ambientes de ulearning e suas estratégias de reconhecimento do perfil dos aprendizes.

\section{- LOCAL (LOcation and Context Aware Learning)}

Barbosa et al., (2008) propõe um modelo que explora sistemas de localização e o suporte à contexto como instrumentos de apoio ao ensino. Os autores apresentam um modelo chamado de LOCAL (LOcation and Context Aware Learning), trata-se de um sistema de localização que acompanha a mobilidade dos aprendizes e, baseado nas suas posições físicas, explora oportunidades educacionais.

Para identificar o perfil dos usuários, o modelo utiliza o padrão PAPI (Public and Private Information for Learners) (PAPI 2006). Basicamente, existem informações persistentes (Contato, Preferências e Interesses) que acompanham o aprendiz independentemente do contexto. Essas informações são armazenadas no Assistente Pessoal (AP) que acompanha o dispositivo móvel. Além disso, há ainda informações contextuais (Relacionamentos, Desempenho e Segurança), as quais estão ligadas aos contextos por onde o aprendiz se desloca.

O LOCAL gerencia ambos os tipos para exploração de oportunidades pedagógicas de acordo com o deslocamento dos alunos. O sistema de localização informa para o tutor, o contexto onde está o aprendiz. O professor e o tutor podem usar essa informação, aliada ao perfil do aprendiz, para determinação dos objetos relevantes no contexto. A proposta deste ambiente evidenciou fortes limitações no que tange às inferências pedagógicas do tutor.

Identificando esse problema, Levis et al., (2008) desenvolveu o PeLeP (Pervasive Learning Profile), cujo propósito foi refinar o rastreamento das atividades de aprendizagem no ambiente baseado em uma análise abrangente do perfil do aprendiz. Para tanto, é utilizado o histórico do aprendiz nos contextos do ambiente ubíquo, durante um determinado período de tempo. São armazenadas informações referentes à localização (contextos no qual o aprendiz esteve), recursos acessados (que objetos de aprendizagem foram acessados), dispositivos utilizados (registra dispositivos como tablet, PC, notebook) e aplicativos utilizados (quais programas foram acessados pelo aprendiz).

Ainda utilizando o LOCAL, Hahn e Barbosa (2008) introduziram uma arquitetura de assistente pessoal e modelado como um agente capaz de explorar automaticamente ambientes de educação ubíqua, com especial cuidado para a detecção e transição de ambientes. O modelo contribuiu na personalização dos processos pedagógicos individuais (através da recomendação de recursos contextualizados e 
relevantes) e em grupo (através do estímulo livre à interação, proporcionado pela análise de similaridade). A principal contribuição do modelo proposto é a organização dos espaços de aprendizagem ubíqua, onde os agentes tem papel de destaque.

\section{- Global}

Explorando uma outra alternativa, Oliveira e Barbosa (2009) apresentaram um modelo de educação ubíqua descentralizado, baseado em sistemas multi-agentes, denominado Global. Este, disponibiliza agentes de software que executam tarefas comuns ao processo de aprendizagem ubíqua. Sendo sua customização direcionada para a criação de novos ambientes de aprendizagem ubíqua, através da extensão desses agentes ou adição de novos.

\section{- CoolEdu}

Trata-se de um ambiente descentralizado de educação ubíqua, Rabello et al., (2012) apresentam um modelo para auxiliar no processo de ensino e aprendizagem através do fomento à colaboração entre os aprendizes de forma contínua. Esta abordagem foi modelada usando agentes e empregando um modelo sócio-interacionista.

A estratégia para reconhecimento do perfil dos aprendizes ocorre por meio de categorias. Dentre elas, há a categoria History que agrupa as informações que o aprendiz está trabalhando, ou já trabalhou. Também é armazenado o contexto onde a dupla interagiu e uma nota, dada pelo próprio aprendiz, referente ao quanto foi útil essa interação. Estes dados podem mudar continuamente e servem como um cache do que está sendo ensinado e aprendido pelo aprendiz, e como um histórico do que já foi ensinado e aprendido.

\section{- UbiGroup}

Em Ferreira, Barbosa e Gluz (2013), os autores propõe um modelo de recomendação ubíqua de conteúdo educacional para grupos de aprendizes, o UbiGroup. Seu objetivo é recomendar Objetos de Aprendizagem (OA) considerando de forma integrada os perfis dos aprendizes e o contexto onde eles estão inseridos. Nesse caso, o modelo conta com um agente Gestor de Perfis (GP) que mantém os perfis dos aprendizes atualizados, realizando ainda um cálculo de similaridade entre eles.

Com base nesse perfil, contexto e a regra de recomendação contida no contexto, o agente define os filtros de pesquisa a serem utilizados e os envia para um agente Comunicador (CO) que efetua a comunicação com os repositórios de OAs. Este agente executa a consulta no repositório e retorna o resultado para o Agente Recomendador (RE). Após a execução das recomendações individuais para os perfis representativos, são unificadas em uma única lista, constituindo uma ordenação prévia de OAs a serem recomendados.

Os ambientes descritos acima, apresentam diferentes estratégias de reconhecimento do perfil dos aprendizes. É preciso reconhecer que mesmo diante das diferentes perspectivas sobre as quais os ambiente ubíquos são destinados, ainda não há uma compreensão cognitiva clara associada aos estilos de aprendizagem existentes. Este fato, gera uma limitação sobre a capacidade desses ambientes alinharem seus requisitos com a perspectiva pedagógica em oferecer um ambiente personalizado. Além de 
ambientes de u-learning, outras estratégias buscam identificar o perfil do aprendiz ubíquo.

Em Mendonça et al., (2014) é apresentado um modelo de arquitetura de software que suporta atividades colaborativas de extensão e enriquecimento de conteúdo digital por meio de anotações e ranqueamento (classificação). Os elementos da arquitetura são capazes de prover informações para serviços de recomendação e personalização da informação no contexto da aplicação, unidos a uma infraestrutura dos ambientes ubíquos de captura e acesso. Esses visam proporcionar aos usuários a reconstituição da experiência vivida em sala de aula, complementando-a com a possibilidade de interações colaborativas e sociais.

Aliança Neto, Neto, e Moreira (2014) apresentam uma abordagem para a construção implícita de perfis de usuário de maneira genérica, independente do domínio de aplicação e facilmente adaptável a sistemas de recomendação de conteúdo. O perfil do usuário representa principalmente seus interesses, que na pesquisa são identificáveis a partir dos conteúdos dos sites acessados pelo estudante.

No momento em que um conteúdo (site, vídeo, rede social, etc.) é acessado, um componente analisador semântico identifica e isola as anotações e/ou marcações já existentes, como HTML e XML. O conteúdo restante é semanticamente anotado com conceitos provenientes das ontologias de domínio. Os dados das anotações semânticas do conteúdo sendo enriquecido são transmitidos para o indexador semântico, que seleciona dados importantes com base nos já cadastrados na ontologia de perfil onde os dados tratados são indexados e armazenados para constituir o perfil individual do usuário.

Em Branquinho et al., (2015), é apresentado uma proposta que tem como objetivo proporcionar um ambiente de computação ubíqua com uma ferramenta que possibilite prover ao usuário um mecanismo para reforço ou alcance de um conjunto de competências. A abordagem faz uso de objetos de aprendizagem adequados ao seu estilo de aprendizagem, que pode ser determinado automaticamente pelo sistema. Na solução do problema de sequenciamento de objetos considerou-se estratégias evolucionárias e uma técnica de busca e, pelos experimentos realizados, chegou-se a resultados melhores fazendo uso de um algoritmo evolucionário steady-state.

É possível observar que além das características nativas dos ambientes ubíquos, estratégias auxiliares são utilizadas no sentido de enriquecer o perfil do aprendiz. Contudo, em ambas as situações é negligenciado o alinhamento sobre a percepção pedagógica.

\section{Estilos de Aprendizagem como Alternativa para Identificar o Perfil do Aprendiz}

Hwang (2006) estabeleceu um conjunto de características de ambientes $u$-learning e que constituem também desafios para designers. Segundo ele, tais ambientes precisam ser: sensíveis ao contexto, fornecer suporte para personalização além de permitir uma aprendizagem no qual e ser capaz de se adaptar temas de conteúdo.

A adaptatibidade desempenha um papel importante na aprendizagem ubíqua, objetivando proporcionar aos aprendizes material de aprendizagem adaptado $\mathrm{e}$ 
personalizado, atividades, e informações no lugar e momento certos (BRANQUINHO et al., 2015). Com isso, essa discussão propõe como alternativa para a identificação perfil do aprendiz ubíquo, o uso de estilos de aprendizagem, permitindo que o ambiente e os materiais nele apresentados se adequem ao perfil e propiciem, assim, um tratamento personalizado.

Existem na literatura diversos modelos de estilos de aprendizagem, contudo pesquisas como Kuljis e Liu (2005) e Carver et al., (1999) apontam que ao se tratar de ambientes educacionais adaptativos, o Modelo de Estilos de Aprendizagem FelderSilverman (do inglês Felder-Silverman Learning Styles Model - FSLSM) descrito em (Felder e Silverman, 1988) pode ser visto como o mais utilizado para classificação de estilos de aprendizagem.

De acordo com o modelo FSLSM, o aluno pode ser caracterizado em quatro dimensões de estilos de aprendizagem. Estas dimensões podem ser identificadas respondendo-se à questionamentos sobre o tipo de informação que o aluno preferencialmente percebe, qual canal sensorial uma informação externa é mais eficientemente, como o aluno prefere processar a informação e como o aluno progride seu entendimento no assunto.

Foram encontradas na literatura algumas abordagens que utilizam o perfil do aluno para permitir uma personalização de ambientes ubíquos. A proposta dessa dicussão é o uso de estilos de aprendizagem para auxiliar na personalização de ambientes de aprendizagem ubíqua.

Relacionar estilos de aprendizagem dos estudantes com ações instrucionais apropriadas é um fator importante ao estímulo da aprendizagem (CHU et al., 2010). Pesquisas têm apontado que a aprendizagem é facilitada se os métodos de ensino utilizados estiverem de acordo com os seus estilos de aprendizagem (GRAF e LIU, 2008; COFFIELD et al., 2009; HAIDER, SINHA e CHAUDHARY, 2010). Além disso, ao identificar o estilo de aprendizagem é possível se beneficiar, obtendo informações sobre como os alunos aprendem, o que lhes proporciona uma compreensão profunda e que tipo de materias frequentemente atendem às suas necessidades (GRAF et al., 2009).

Tal proposta, está alinhada à pesquisas como Graf et al., (2008), Kinshuk et al., (2009) e Graf et al., (2009), no qual identificam que a aprendizagem é facilitada se as estratégias pedagógicas estiverem de acordo com o estilo de aprendizagem do aprendiz, tornando o processo de aprendizagem efetivo e melhorando consideravelmente sua performance.

Propostas para detecção automática de estilos de aprendizagem (GRAF e VIOLA, 2009; LIMONGELLI et al., 2009) têm sido exploradas. Elas utilizam sistemas de inferência determinísticos baseados em padrões de comportamento pré-definidos do aprendiz, de forma que, através de seu comportamento e ações no sistema, possam inferir seus estilos de aprendizagem.

Contudo, tais abordagens ignoram importantes considerações relacionadas aos aspectos dinâmicos e não-determinísticos de estilos de aprendizagem e do comportamento do aprendiz. Nesse contexto, Dorça et al., (2014) apresentou uma abordagem automática, dinâmica e probabilística para modelagem de estilos de aprendizagem, avaliando e comparando através de experimentos, três diferentes políticas de aprendizagem por reforço. 
Paredes et al., (2005) também proporam a utilização de modelos representados através de valores probabilísticos. Os autores apresentam a concepção e implementação de um sistema centralizado com uma abordagem baseada em múltiplos modelos para apoiar a personalização de ambientes de aprendizagem ubíqua. A arquitetura do sistema é baseada em informações sobre o aplicativo, características do usuário, comportamento e o ambiente no qual ele está inserido. Ao final, é gerado uma estereótipo a partir das características estabelecidas no modelo.

Chiou e Tseng (2012) propõe uma navegação personalizada e sensível no contexto u-learning baseada em estilos de aprendizagem. Os autores utilizaram a Teoria da Orientação da Aprendizagem (Learning Orientation Theory) como estratégia para reconhecer os estilos de aprendizagem dos alunos. Tal teoria leva em consideração:

i. A influência de um conjunto abrangente de fatores psicológicos que influenciam a aprendizagem;

ii. O impacto das emoções e intenções na aprendizagem;

iii. As relações entre os aprendizes nos ambientes de aprendizagem e a capacidade de aprendizagem;

iv. A perspectiva abrangente para projetar ambientes para suporte à aprendizagem.

Existem estudos que utilizam técnicas de aprendizagem supervisionada de máquina, tais como redes neurais e redes Bayesianas (Carmona e Castillo, 2008; Zatarain-Cabada et al., 2009). A dificuldade dessas abordagens é a elevada complexidade de implementação e grande consumo de recursos computacionais de forma que a presença simultânea de vários estudantes pode comprometer, ou mesmo inviabilizar seu uso.

Apesar da literatura apresentar várias iniciativas no que diz respeito à identificação de perfis, ainda existem algumas lacunas a serem preenchidas para a melhor prática ensino. Faz-se necessário reforçar que em um cenário ubíquo, o perfil do aprendiz constitui-se um elemento fundamental, em função das inúmeras possibilidades de interatividade e as diversas tecnologias de acesso a informação. Desta forma, torna-se necessário que o ambiente conheça em profundidade o aprendiz e o auxilie a interagir com a diversidade de elementos do ambiente e oportunidades de interação à disposição.

\section{Considerações Finais}

Este trabalho, conforme descrito em sua introdução, procura evidenciar a importância de considerar a adaptabilidade nos ambientes de aprendizagem ubíquos. Para isso foram apresentados estudos que já apontam alternativas para a personalização desses ambientes, bem como, quais procedimentos foram utilizados.

Diversas iniciativas seja na construção de ambientes ou na proposição de estratégias têm oferecido diferentes caminhos para uma compreensão e atendimento às necessidades dos aprendizes. É importante destacar que cada iniciativa possui sua particularidade e nos casos descritos percebe-se um desalinhamento entre o que é desenvolvido com o potencial pedagógico que pode ser explorado. 
Considerando que dentro do contexto de aprendizagem ubíqua a adaptabilidade e a flexibilidade são pontos chaves para um melhor entendimento das reais necessidades dos aprendizes, um ponto desafiador que permeia essa discussão concentra-se na percepção sobre o perfil dos aprendizes. Entre os pontos apresentados, compreender o perfil do aprendiz, levando em consideração aspectos complementares associados ao seu estilo de aprendizagem sinaliza como uma oportunidade a ser explorada neste campo.

\section{Agradecimento}

Luma da Rocha Seixas é bolsista de doutorado da Fundação de Amparo a Ciência e Tecnologia de Pernambuco - FACEPE - Processo Nº. PBPG-0540-1.03/15.

\section{Referências}

Barbosa, D. N. F. (2007). Um Modelo de Educação Ubíqua Orientado à Consciência do Contexto do Aprendiz. Tese de Doutorado, Unisinos.

Barbosa, C.F.R, Geyer, J.L.V. Barbosa. (2005) GlobalEdu: An Architecture to Support Learning in a Pervasive Computing Environment. In IFIP Workshop on Educational Technology, Perth, páginas 1-10.

Barbosa, J. L. V., Hahn, R. M., Rabello, S., Barbosa, D. F. N. (2008) LOCAL: a Model Geared Towards Ubiquitous Learning. In 39th ACM Technical Symposium on Computer Science Education (SIGCSE). New York, páginas 432-436,ACM Press

Branquinho, A., Lopes, C., Dorça, F., Fernandes, M., Cattelan, R., and de Ávila, A. J. N. (2015). Aquisição Automática de Competências num Ambiente Educacional Ubíquo. In Anais do Simpósio Brasileiro de Informática na Educação (Vol. 26, No. 1, p. 1207).

Bra, P. De and Calvi, L. AHA! (1998) An open adaptive hypermedia architecture. New Review of Hypermedia and Multimedia, vol. 4, no. 1, pp. 115-139, 1998.

Brusilovsky, P., Eklund, J. and Schwarz, E. (1998). Web-based education for all: A tool for developing adaptive courseware. in Seventh International WWW Conference, pp. 291-300, Elsevier Science B.V.

Carver, C. A., Howard, R.A. , and Lane W.D. (1999). Addressing different learningstyles through course hypermedia. IEEE Transactions on Education, 42(1), 33-38.

Carmona, C. e Castillo, G. (2008). Designing a Dynamic Bayesian Network for Modeling Students Learning Styles. In Eighth IEEE International Conference on Advanced Learning Technologies, pages 346-350. IEEE.

Chen, C.; Li, Y. (2010). Personalised context-aware ubiquitous learning system for supporting effective English vocabulary learning. Interactive Learning Environments, v. 18, n. 4 , p. $341-364$.

Chiou, C. K., and Tseng, J. C. (2012). Design of a personalized navigation support system for context-aware ubiquitous learning environment. In Proceedings of the 2012 RecSys workshop on Personalizing the local mobile experience (pp. 1-6). ACM. 
de Aliança Neto, A. S., Neto, F. M. M., \& Moreira, J. D. (2014). Uma Nova Abordagem para o Perfilamento de Usuários em Ambientes de Aprendizagem Ubíqua. In Anais do Simpósio Brasileiro de Informática na Educação (Vol. 25, No. 1, p. 1243).

Dorça, F. A., Lima, L. V., Fernandes, M. A., and Lopes, C. R. (2014). Avaliação de Políticas de Aprendizagem por Reforço para Modelagem Automática e Dinâmica de Estilos de Aprendizagem: Uma Análise Experimental. Revista de Informática Aplicada, 9(2).

Durán, Elena B., Margarita M. Álvarez, and Silvina I. Únzaga (2014). Ontological model-driven architecture for ubiquitous learning applications."Proceedings of the 7th Euro American Conference on Telematics and Information Systems. ACM.

Felder, R. M.; Silverman, L. K. (1988) Learning and teaching styles in engineering education. Engineering education, v. 78, p. 674-681.

Ferreira, L. G. A., Barbosa, J. L. V., and Gluz, J. C. (2013). Um Modelo de Recomendação Ubíqua de Conteúdo para Grupos de Aprendizes. In Anais do Simpósio Brasileiro de Informática na Educação (Vol. 24, No. 1, p. 697).

Gasperini, M. A. I., Amaral, Pimenta, M. S., Oliveira, J. P. M. (2004) Navegação Adaptativa no Ambiente de EAD AdaptWeb: uso de XML na Adequação de Conteúdo e Navegação. In III Congresso Internacional de Ergonometria e Usabilidade Design de Interfaces e Interação Humano-Computador, páginas 124-130.

Graf, S., Kinshuk, and Liu, T. (2009). Supporting teachers in identifying students' learning styles in learning management systems: An automatic student modelling approach.Journal of Educational Technology \& Society, 12(4):3-14.

Graf, S., Liu, T.-C., and Kinshuk (2008). Interactions Between Students Learning Styles, Achievement and Behaviour in Mismatched Courses. In Proceedings of the International Conference on Cognition and Exploratory Learning in Digital Age (CELDA 2008), pages 223-230. IADIS International Conference.

Graf, S.,; Viola, S. (2009). Automatic student modelling for detecting learning style preferences in learning management systems. Citeseer.

Hahn, R. M., e Barbosa, J. L. V. (2008). Uma Arquitetura de Assistente Pessoal Orientada a Ambientes de Aprendizagem Ubíqua. In Anais do Simpósio Brasileiro de Informática na Educação (Vol. 1, No. 1, pp. 736-745).

Hwang G. (2006)Criteria and Strategies of Ubiquitous Learning. Proceedings of the IEEE International Conference on Sensor Networks, Ubiquitous, and Trustworthy Computing (SUTC'06), IEEE.

Kinshuk, Liu, T., and Graf, S. (2009). Coping with Mismatched Courses: Students' behaviour and performance in courses mismatched to their learning styles. Educational Technology Research and Development, 57(6):739-752.

Kong, S. C., Chan, T. W., Griffin, P., Hoppe, U., Huang, R., Kinshuk, and Yu, S. (2014). E-learning in school education in the coming 10 years for developing 21st century skills: Critical research issues and policy implications. Journal of Educational Technology \& Society, 17(1), 70-78. 
Kuljis, J.; Liu, F. (2005) A Comparison of Learning Style Theories on the Suitability for elearning. Web Technologies, Applications, and Services, p. 191-197, 2005.

Levis, D., Barbosa, J. L., Pinto, S. C. C. S., and Barbosa, D. (2008). Aperfeiçoamento automático do perfil do aprendiz em ambientes de educação ubíqua. Revista Brasileira de Informática na Educação, 16(1), 29-41.

Limongelli, C., Sciarrone, F., Temperini, M., and Vaste, G. (2009). Adaptive learning with the LS-plan system: a field evaluation. Learning Technologies, IEEE Transactions on, 2(3), 203-215.

Mendonça, I. E. S., Araujo, R. D., Mendes, M. M., Brant-Ribeiro, T., Dorça, F. A., \& Cattelan, R. G. (2014). Explorando Funcionalidades Sociais e Colaborativas em Ambientes Educacionais Ubiquos.

Ogata, H., Yin, C., Yano, Y. (2005). JAPELAS: Support-ing Japanese Polite Expressions Learning Using PDA towards Ubiquitous Learning. The Journal of Information and Systems in Education. 2(1): 33-39,

PAPI (2006). "Draft standard for learning technology. public and private information (papi) for learners (papi learner)", August.

Paredes, R. G. J., Ogata, H., Yano, Y. San Martin, G. A. (2005). A Multi-Model Approach for Supporting the Personalization of Ubiquitous Learning Applications.International Workshop on Wireless and Mobile Technologies in Education (WMTE'05). IEEE.

Peng, H.i; Chou, C.; Chang, C. (2008) From virtual environments to physical environments: Exploring interactivity in ubiquitous-learning systems. Journal of Educational Technology \& Society, v. 11, n. 2, p. 54-66.

Rabello, S., Barbosa, J. L., Oliveira, J., Wagner, A., Barbosa, D. N., and Bassani, P. B. (2012). Um Modelo para Colaboração em Ambientes Descentralizados de Educação Ubíqua. In Anais do Simpósio Brasileiro de Informática na Educação (Vol. 23, No. 1).

Tsai, Chin-Chung. (2010) Conceptions of learning versus conceptions of web-based learning: The differences revealed by college students. Computers \& Education, v. 53 , n. 4 , p. 1092-1103.

$\mathrm{Wu}, \mathrm{H}$., Kort, E. e Bra, P. de. (2001) Design issues for general-purpose adaptive hypermedia systems. in Proceedings of the 12th ACM Conference on Hypertext and Hypermedia, pp. 141-150, ACM.

Zakaria, M. R. and Brailsford, T. J. (2002).User Modelling and Adaptive Educational Hypermedia Frameworks for Education. New Review of Hypermedia and Multimedia, vol. 1, no. 8, pp. 83-97,

Zatarain-Cabada, R., Barrón-Estrada, M., Zepeda-Sánchez, L., Sandoval, G., OsorioVelazquez, J., e Urias-Barrientos, J. (2009). A Kohonen Network for Modeling Students' Learning Styles in Web 2.0 Collaborative Learning Systems. MICAI 2009: Advances in Artificial Intelligence, pages 512-520. 